\title{
Metadoxine Versus Placebo for the Treatment of Non-alcoholic Steatohepatitis: A Randomized Controlled Trial
}

\author{
Kotacherry T. Shenoy ${ }^{* \dagger}$, Leena K. Balakumaran ${ }^{\dagger}$, Philip Mathew ${ }^{\ddagger}$, Mohan Prasad $^{\S}$, Boddu Prabhakar" ${ }^{\|}$Ajit Sood ${ }^{\pi}$, \\ Shivaram P. Singh ${ }^{\#}$, Nagaraj P. Rao**, Shoukat A. Zargar ${ }^{\dagger \dagger}$, Angelo A. Bignamini ${ }^{\ddagger \ddagger}$ \\ "Sree Gokulam Medical College and Research Foundation, Trivandrum, ${ }^{\dagger}$ Population Health and Research Institute, Trivandrum, ${ }^{\ddagger} P V S$ Memorial \\ Hospital, Cochin, ${ }^{\S}$ G. Hospital \& Post Graduate Institute, Coimbatore, "Osmania Medical College \& Hospital, Hyderabad, "Dayanand Medical

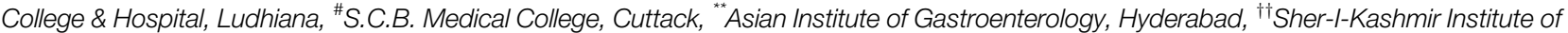 \\ Medical Sciences, Kashmir, India and ${ }^{\ddagger}$ School of Specialization in Hospital Pharmacy, University of Milan, Italy
}

\begin{abstract}
Objective and design: The study aimed at assessing the therapeutic efficacy and safety of metadoxine versus placebo on the ultrasonographic and histological features of non-alcoholic steatohepatitis (NASH). Subjects: 134 subjects with biopsy-confirmed NASH were randomized to receive metadoxine $500 \mathrm{mg}$ two times daily $(n=75)$ or placebo $(n=59)$ added to the standard of care, over 16 weeks. Efficacy endpoints: Originally, the primary efficacy endpoint was the composite of: reduction in the steatosis by $\geq 1$ grade, reduction in hepatic necro-inflammation by $\geq 1$ grade and ALT normalization. Since $>50 \%$ of patients refused the second biopsy, it was decided to analyze only the individual parameters. Results: There was no significant difference between the treatment and the placebo groups in either liver histology or ALT or AST. Overall, as expected both groups showed reduction in serum ALT and AST compared to baseline. Compared to placebo (9 out 54), patients on metadoxine (34 out of 75) had significantly higher rates of improvement in 1-point in steatosis grade on ultrasound $(P$-value $<0.001)$. Safety and tolerability did not differ between treatments. Conclusion: Metadoxine is not effective in improvement of liver histology or serum ALT or AST in patients with NASH. However, there was significant improvement of steatosis assessed by ultrasound. To properly estimate the effects on histology and transaminases, further studies of longer duration and at higher doses are needed. (J Clin Exp Hepatol 2014;4:94-100)
\end{abstract}

$\mathrm{N}$ on-alcoholic fatty liver disease (NAFLD) is an adaptive response of the liver to insulin resistance. It is an increasingly common condition and has high prevalence among those with obesity and diabetes. ${ }^{1}$ A meta-analysis of 23 randomized controlled trials in non-alcoholic steatohepatitis (NASH) or NAFLD ${ }^{2}$ showed that most RCTs were small and did not exceed 1-year duration. Thiazolidinediones improved steatosis and inflammation but yielded significant weight gain. Randomized clinical trials (RCTs) with antioxidants had issues related

Keywords: NASH, metadoxine, controlled clinical trial Received: 8.1.2014; Accepted: 3.3.2014; Available online: 27.3.2014 Address for correspondence: Prof. Kotacherry T. Shenoy, Sree Gokulam Medical College and Research Foundation Trivandrum India.

E-mail: dr.ktshenoy@gmail.com

Abbreviations: ALD: alcoholic liver disease; ALT: alanine transaminase; ANCOVA: analysis of covariance; AST: aspartate transaminase; ATP: adenosine triphosphate; GGT: gamma-glutamyl transferase; GSH: glutathione; HOMA-IR: homeostasis model assessment for insulin resistance; ITT: intention to treat; MRI: magnetic resonance imaging; MRS: MR spectroscopy; NADH: nicotinamide adenine dinucleotide; NAFLD: nonalcoholic fatty liver disease; NASH: non-alcoholic steatohepatitis; PDFF: proton-density fat-fraction; RCTs: randomized clinical trials; RIQ: range interquartile; TNF: tumor necrosis factors

http://dx.doi.org/10.1016/j.jceh.2014.03.041 to type and dose of drug, duration and implementation of lifestyle intervention. Pentoxifylline, telmisartan and L-carnitine improved liver histology in at least one RCT in NASH. Vitamin E was superior to placebo for the treatment of non-alcoholic steatohepatitis in adults without diabetes. In a recent meta-analysis, it has been shown that pioglitazone improves steatosis, ballooning degeneration, and lobular inflammation and there is a suggestion that it may also improve fibrosis when pioglitazone was analyzed alone. ${ }^{3}$ In a recent systematic review of pentoxifylline in NAFLD/NASH, it was found that the drug reduced AST and ALT levels and may improve liver histology in patients with NAFLD/NASH, but did not appear to affect the cytokines. ${ }^{4}$

Metadoxine (pyridoxine-L-2-pyrrolidone-5-carboxylate) exhibits multifactorial pharmacological properties suited for its utilization in NAFLD, NASH, and alcoholic liver disease (ALD). These include restoration of nicotinamide adenine dinucleotide (NADH), glutathione (GSH) and adenosine triphosphate (ATP) levels, as well as the proportion between saturated and unsaturated fatty acids and esters in the liver, and reduces oxidative stress. ${ }^{5-9}$ Metadoxine also decreased the synthesis of fibronectin and procollagen and the activity of proline hydroxylase in the liver after 
$\mathrm{CCl}_{4}$ challenge. ${ }^{10,11}$ In hepatic stellate cells, metadoxine prevented the increase in collagen and attenuated tumor necrosis factors (TNF)-alpha secretion caused by acetaldehyde. ${ }^{12}$ Thus, metadoxine appears to be a potentially effective strategy to manage non-alcoholic steatohepatitis and non-alcoholic fatty liver disease (NAFLD). Based on experimental and early clinical studies, ${ }^{13}$ it has been hypothesized that metadoxine may be useful in NASH and NAFLD. Since, however, clinical investigations were so far focused on alcoholic liver disease, ${ }^{14}$ we designed this study to monitor whether similar results could be obtained in NASH.

\section{METHODS}

The study was designed as a randomized, placebocontrolled, double-blind, multicentre trial in parallel groups of patients. We enrolled patients of either sex, aged between 18 and 60 years, drinking <20 g/day of ethanol, with obesity, upper abdominal symptoms, dyspepsia or elevated transaminases. Liver biopsy and ultrasound scan of the abdomen were performed to confirm the presence of NASH, defined as association of steatosis and hepatocyte ballooning with a pattern of centrilobular accentuation or association of steatosis, hepatocyte ballooning, and perisinusoidal fibrosis with a pattern of centrilobular accentuation. Patients with advanced liver disease, taking >20 g/day of ethanol, positive for hepatitis $\mathrm{B}$ or $\mathrm{C}$, under established medications known to cause steatosis, with renal failure or who had recently used the test medication were not recruited. All patients received the standard of care each center normally applied to patients with NASH (individualized nutritional counseling for adequate caloric intake and appropriate lifestyle modifications, though there was no specific dietary prescription or exercise across centers). In addition, after signing the informed consent, the patients were randomly assigned to receive the test treatment (metadoxine $500 \mathrm{mg}$ tablets) or matching placebo twice daily for 16 weeks, followed by 4 -week untreated monitoring. The study statistician prepared a computer-generated randomization list, which was kept blinded until the end of the statistical analysis. No emergencies occurred that required breaking the blind.

Patients were monitored at 4-week intervals from randomization to week 20 . Clinical history and physical examination were completed at each visit, as were hematology, hematochemistry, compliance and adverse events except at week 20. Laboratory tests included liver enzymes, complete blood count and prothrombin time. There was no centralized laboratory; however all the centers were using similar auto analyzers and standards for normal values. Fasting insulin and fasting glucose were used to calculate insulin resistance according to the homeostasis model assessment technique (HOMA-IR) at baseline. We scored the extent of steatosis at screening and end of the study (as $0=$ absent, $1=$ mild, $2=$ moderate, $3=$ severe) by the difference in echo amplitude between liver and kidney and the loss of echoes from the walls of the portal veins and/or gall bladder wall. ${ }^{15,16}$

The liver pathologists at each center determined the presence and severity of histological diagnosis of NASH at baseline and end of treatment. The study pathologists confirmed the adequacy of the liver biopsy specimens for evaluation. We graded and staged the histological conditions of the biopsies according to Brunt et al. ${ }^{17}$ Necroinflammation was graded as $0=$ absent; $1=$ mild, occasional ballooned hepatocytes, scattered and mild lobular inflammation, no or mild portal inflammation; 2 = moderate, obvious hepatocyte ballooning, mild lobular inflammation, mild to moderate portal inflammation; 3 = severe, marked hepatocyte ballooning, scattered lobular inflammation and polymorphonuclear cells, mild to moderate portal inflammation. Fibrosis was staged as $0=$ no fibrosis; 1 = zone 3 perisinusoidal fibrosis, focal or extensive; 2 = perisinusoidal fibrosis, focal or extensive, and periportal fibrosis, focal or extensive; 3 = bridging fibrosis, focal or extensive; grade 4 cirrhosis.

Using the criteria for steatosis by ultrasound scan, radiologists interpreted the findings at each center as this could not be centralized.

We originally set as primary endpoint the composite of: reduction in steatosis by $\geq 1$ grade, reduction in necroinflammation by $\geq 1$ grade and ALT normalization, however, after closing the study and in view of the small number of patients suitable for such analysis, we decided to analyze only the individual components. The rationale for the primary endpoint was based on the placebo effect observed in clinical trials. $15-33 \%$ of subjects receiving placebo showed 1-point improvement in steatosis, ballooning degeneration, lobular inflammation, NASH fibrosis and combined inflammation scores. ${ }^{18}$

\section{Statistics}

Based on the information from the studies in AFLD, we anticipated a difference of 30 percent points with the test medication over the placebo effect. Accounting for an expected loss of information (refused second biopsy) of approximately $50 \%$ and retaining a total alpha error $\leq 0.05$, a sample of approximately 200 patients would have had $80 \%$ power to detect the anticipated difference with $P$ $\leq 0.025$ two-tailed for each correlated analysis, in the intention to treat (ITT) population (all randomized patients) and in the efficacy population (only patients with valid final measurement).

Normally distributed measures were summarized as mean and standard deviation (SD) and compared with the independent samples $t$-test or, where appropriate, with the analysis of covariance (ANCOVA) for repeated measures, using treatment and sex as fixed factors, center 
Table 1 Baseline Profile of Patients.

\begin{tabular}{|c|c|c|}
\hline & Metadoxine, $N=75$ & Placebo, $N=59$ \\
\hline Sex: males, $N(\%)$ & $52(69.3)$ & 43 (72.9) \\
\hline Age; years (mean $\pm \mathrm{SD}$ ) & $39.8 \pm 10.4$ & $41.1 \pm 8.5$ \\
\hline $\begin{array}{l}\mathrm{BMI}, \mathrm{kg} / \mathrm{M}^{2} \\
(\mathrm{mean} \pm \mathrm{SD})\end{array}$ & $26.8 \pm 3.8$ & $27.0 \pm 3.9$ \\
\hline Normal, $N(\%)$ & $11(14.7)$ & $6(10.2)$ \\
\hline Overweight, $N(\%)$ & $13(17.3)$ & $15(25.4)$ \\
\hline Obese, $N(\%)$ & $51(68.0)$ & $38(64.4)$ \\
\hline $\begin{array}{l}\text { Waist circumference, } \\
\mathrm{cm}(\text { mean } \pm \mathrm{SD})\end{array}$ & $92.9 \pm 8.8$ & $93.0 \pm 7.2$ \\
\hline Domicile: rural, $N(\%)$ & $53(70.7)$ & $41(69.5)$ \\
\hline $\begin{array}{l}\text { ALT, U/L } \\
\text { (median [RIQ]) }\end{array}$ & $80.0[55.5,128.0]$ & $83.5[55.5,129.3]$ \\
\hline $\begin{array}{l}\text { AST, U/L } \\
\text { (median [RIQ]) }\end{array}$ & $54.0[38.0,76.5]$ & $62.0[38.0,80.5]$ \\
\hline $\begin{array}{l}\text { Alkaline phosphatase. } \\
\mathrm{U} / \mathrm{L} \text { (median [RIQ]) }\end{array}$ & $112.0[71.5,187.5]$ & $107.0[69.5,175.5]$ \\
\hline $\begin{array}{l}\text { GGT, U/L } \\
\text { (median [RIQ]) }\end{array}$ & $46.5[32.0,64.4]$ & $43.2[34.1,52.5]$ \\
\hline $\begin{array}{l}\text { Bilirubin, mg/dL } \\
\text { (median [RIQ]) }\end{array}$ & $0.86[0.60,1.15]$ & $0.80[0.60,1.39]$ \\
\hline $\begin{array}{l}\text { Fasting glucose, } \\
\mathrm{mg} / \mathrm{dL} \text { (mean } \pm \mathrm{SD} \text { ) }\end{array}$ & $100.6 \pm 28.6$ & $102.8 \pm 31.5$ \\
\hline $\begin{array}{l}\text { Serum insulin, } \\
\mathrm{mU} / \mathrm{L} \text { (median [RIQ]) }\end{array}$ & $11.2[8.2,18.3]$ & $10.8[6.1,17.8]$ \\
\hline $\begin{array}{l}\text { HOMA-IR } \\
\text { (median [RIQ]) }\end{array}$ & $2.58[1.81,4.68]$ & $2.26[1.43,5.84]$ \\
\hline $\begin{array}{l}\text { HOMA- } \beta \text { function } \\
\text { (median [RIQ]) }\end{array}$ & $125.6[103.1,186.8]$ & $107.8[66.5,258.1]$ \\
\hline $\begin{array}{l}\text { Steatosis, } \\
N(\%): \text { absent }\end{array}$ & $11(14.7)$ & $13(22.0)$ \\
\hline Mild & $16(21.3)$ & 20 (33.9) \\
\hline Moderate & $46(61.3)$ & $24(40.7)$ \\
\hline Severe & $2(2.7)$ & $2(3.4)$ \\
\hline
\end{tabular}

Necro-inflammatory grade, $N(\%)$ :

\begin{tabular}{|c|c|c|}
\hline 0 & $1(1.3)$ & $0(0.0)$ \\
\hline 1 & $26(34.7)$ & $21(35.6)$ \\
\hline 2 & $29(38.7)$ & $27(45.8)$ \\
\hline 3 & $18(24.0)$ & 11 (18.6) \\
\hline Undetermined & $1(1.3)$ & $0(0.0)$ \\
\hline \multicolumn{3}{|c|}{ Fibrosis stage, $N(\%)$ : } \\
\hline 0 & $34(45.3)$ & $29(49.2)$ \\
\hline 1 & $16(21.3)$ & $13(22.0)$ \\
\hline 2 & $20(26.7)$ & $14(23.7)$ \\
\hline 3 & $4(5.3)$ & $3(5.1)$ \\
\hline Undetermined & $1(1.3)$ & $0(0.0)$ \\
\hline
\end{tabular}

BMI: body mass index; ALT: alanine transaminase; AST: aspartate transaminase; GGT: gamma-glutamyl transferase; HOMA-IR: homeostasis model assessment for insulin resistance computed as [glucose (mg/ $\mathrm{dL}) \times$ insulin $(\mathrm{mU} / \mathrm{L})] / 405$; RIQ: range interquartile (25th, 75th percentile). as random factor and baseline value and age as covariates. Non-normally distributed measures were summarized as median and interquartile range (RIQ: 25th and 75th percentile) and compared with the Mann-Whitney $U$ test. Nominal variables were presented as counts and proportion and compared with the chi-square test. In the ITT analysis, we replaced the changes in ultrasonographic steatosis score, necro-inflammation score and fibrosis staging with the baseline value for all those patients without valid final data; the patient with uninterpretable baseline biopsy was classified as "failure" in the outcome analysis and the score change from baseline was set as zero. All analyses were performed with SPSS (version 17).

The study was performed in compliance with the Declaration of Helsinki and was approved by the Ethics Committees at all involved institutions.

\section{RESULTS}

After three-year of recruitment (February 2008 to January 2011), in view of the difficulty to recruit patients willing to undergo two liver biopsies within less than 6 months, the steering committee interrupted the study, when 134 patients had been enrolled in the 8 participating centers (59 randomized to placebo and 75 to the test medication). The baseline characteristics were similar in the two groups (Table 1). Figure 1 summarizes the patients' disposition and reports the number of patients considered in the ITT analysis and in the efficacy population analysis.

Table 2 summarizes the outcomes observed on steatosis, necro-inflammation, fibrosis, and transaminases. Significantly more metadoxine-treated patients exhibited improved grading of steatosis at ultrasonography, in comparison with those randomized to placebo, in both the ITT and the efficacy population $(P<0.001)$. In the ITT analysis, 34 of 75 patients in the metadoxine group $(45.3 \%$; $95 \%$ CI: $33.8 \%-57.3 \%)$ exhibited improvement, compared to 9 out of 59 in the placebo group (15.3\%; 95\% CI: $7.2 \%-27.0 \%)$. The level of significance of this difference $(P=0.0002)$ largely exceeded the critical value set in the study set-up $(P \leq 0.025$; Figure 2). Patients treated with metadoxine had an odds ratio to experience reduced steatosis of 4.61 (95\% CI: 1.98-10.70), corresponding to a Number Needed to Benefit of 4 (95\% CI: 3-7). The variation in the necroinflammatory grade and fibrosis stage among the patients with initial and final biopsies was small and did not significantly differ across treatment groups.

ALT and AST significantly decreased over time $(P=0.001$ and $P=0.014$, respectively, repeated measures ANCOVA, Greenhouse-Geisser statistic, Figure 3) among the patients with all measures available. The extent of change, however, was not significantly different between groups among the patients with baseline and final measurements.

During the trial, no deaths nor serious or clinically relevant adverse events occurred. We monitored a number of 


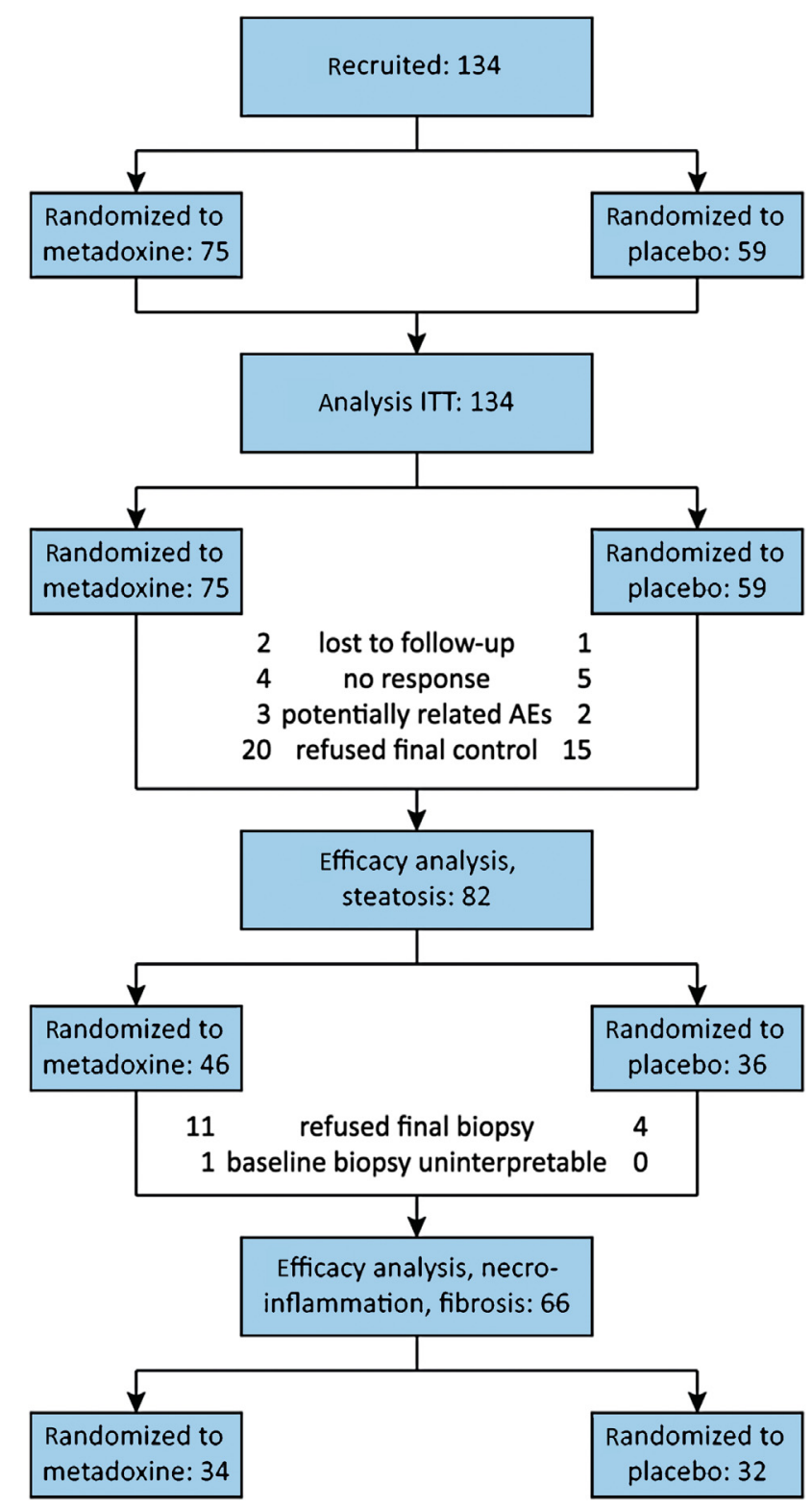

Figure 1 Patients disposition and number of patients considered in each analysis. ITT, intention to treat.

potential adverse events, 30 in the test group (average: 0.025 per patient-week) and 13 among the controls (average: 0.014 per patient-week), however, only in three patients the events were classified as potentially treatment-related. Among the metadoxine-treated patients, we observed one patient reporting severe epigastric pain, and two reporting moderate dyspepsia with epigastric pain. Among the controls, we observed one patient with moderate headache and one with moderate epigastric pain and dyspepsia. During observation, there were no clinically relevant abnormalities that could not be explained be the underlying pathology, nor clinically or statistically significant changes in laboratory tests, vital signs or physical findings (data not shown).

\section{DISCUSSION}

There are very few published reports on the prevalence of NAFLD/NASH from India, ${ }^{19-23}$ information of possible pharmacological treatments is scanty, and this study may help to partly fill this gap. Moreover, the clinical trials on the various compounds in NAFLD/NASH yielded conflicting results with no benefit to partial improvement in steatosis and necro-inflammatory grade and fibrosis staging. ${ }^{2}$

In this multisite, double-blind, randomized, placebocontrolled study in NASH, we evaluated the efficacy of metadoxine versus placebo in eight clinical centers in India. In this study, we observed a significant decrease of ALT and AST without differences between groups. This can possibly indicate that the initial response of transaminases to the standard of care is favorable. However, we also noted that the improvement is almost completely concentrated in the initial 4-week period, suggesting that, to obtain normalization of the enzyme levels, normalization of liver histopathology should be achieved first.

MRI is the gold standard for fat quantification in NASH trials. Magnetic resonance imaging (MRI) technique, the proton-density fat-fraction (PDFF), as well as by conventional MR spectroscopy (MRS) can detect small changes in liver fat and they may be better than histology to detect longitudinal changes in hepatic fat in NASH. ${ }^{24,25}$ Since MRI and MRS were not available across all the trial sites, ultrasound was used for imaging derived steatosis assessment.

In our trial we observed only a limited improvement of necro-inflammation grade and practically no improvement of fibrosis stage, with only $26 \%$ and respectively, $14 \%$ of patients showing a measurable improvement. This finding on the one side prevents estimating a true difference between treatments, if any, but on the other indicates that the length of observation is probably too short in relation to the potential progression of the histologic liver condition.

The duration of observation is, indeed, the major limitation of the study. It may be considered responsible for not observing changes in histopathology, but it is also the major reason for the decision to terminate the trial before completion. Though we planned to have repeat biopsy in all those randomized, a significant number of patients did not agree for the repeat biopsy at the end of this study and hence, we did not have sufficient number with the ultrasound scan and liver histology to arrive at the treatment efficacy and this is a limitation in the present study. As anticipated, among those who did accept to participate, the proportion of patients refusing the second biopsy was as high as $51 \%$ but-and this was not expectedthe refusal of the second biopsy in several patients included also the refusal to undergo the second, non-invasive, ultrasonography. Thus, future trials should be planned with a longer duration of observation, probably up to one year, 
Table 2 Study Outcomes.

\begin{tabular}{|c|c|c|c|c|c|c|c|}
\hline \multirow[t]{2}{*}{ Outcome } & \multirow[t]{2}{*}{ Measure } & \multicolumn{3}{|c|}{ Efficacy population } & \multicolumn{3}{|c|}{ ITT population } \\
\hline & & Metadoxine & Placebo & $\boldsymbol{P}$ & Metadoxine & Placebo & $\boldsymbol{P}$ \\
\hline \multirow{2}{*}{$\begin{array}{l}\text { Steatosis } \\
\text { grade }\end{array}$} & Mean change \pm SD (N) & $-0.76 \pm 0.92(46)$ & $0.00 \pm 0.76(36)$ & $<0.001^{a}$ & $-0.08 \pm 1.12(75)$ & $0.37 \pm 0.76(59)$ & $0.006^{a}$ \\
\hline & $\begin{array}{l}\text { Improved patients, } \\
n / N(\%[95 \% \mathrm{CI}])\end{array}$ & $\begin{array}{l}34 / 46(73.9 \% \\
[58.9-85.7])\end{array}$ & $\begin{array}{l}9 / 36(25.0 \% \\
[12.1-42.2])\end{array}$ & $<0.001^{\mathrm{b}}$ & $\begin{array}{l}34 / 75(45.3 \% \\
[33.8-57.3])\end{array}$ & $\begin{array}{l}9 / 59(15.3 \% \\
[7.2-27.0])\end{array}$ & $<0.001^{\text {b }}$ \\
\hline \multirow{2}{*}{$\begin{array}{l}\text { Necro- } \\
\text { inflammation } \\
\text { grade }\end{array}$} & Mean change \pm SD $(\mathrm{N})$ & $-0.71 \pm 0.76(34)$ & $-0.50 \pm 0.67(32)$ & $0.249^{a}$ & $0.09 \pm 0.95(75)$ & $0.10 \pm 0.87(59)$ & $0.965^{a}$ \\
\hline & $\begin{array}{l}\text { Improved patients, } \\
n / N(\%[95 \% \mathrm{Cl}])\end{array}$ & $\begin{array}{l}20 / 34(58.8 \% \\
[40.7+75.4])\end{array}$ & $\begin{array}{l}15 / 32(46.9 \% \\
[29.1-65.3])\end{array}$ & $0.331^{\mathrm{b}}$ & $\begin{array}{l}20 / 75(26.7 \% \\
[17.1-38.1])\end{array}$ & $\begin{array}{l}15 / 59(25.4 \% \\
[15.0-38.4])\end{array}$ & $0.871^{b}$ \\
\hline \multirow{2}{*}{$\begin{array}{l}\text { Fibrosis } \\
\text { stage }\end{array}$} & Mean change $\pm \mathrm{SD}(\mathrm{N})$ & $-0.24 \pm 0.86(34)$ & $-0.16 \pm 0.72(32)$ & $0.687^{a}$ & $0.38 \pm 0.84(75)$ & $0.36 \pm 0.78$ (59) & $0.875^{a}$ \\
\hline & $\begin{array}{l}\text { Improved patients, } \\
\text { n/N (\% [95\% Cl]) }\end{array}$ & $\begin{array}{l}12 / 34(35.3 \% \\
[19.7-53.5])\end{array}$ & $\begin{array}{l}7 / 32(21.9 \% \\
[9.3-40.0])\end{array}$ & $0.229^{b}$ & $\begin{array}{l}12 / 75(16.0 \% \\
[8.6-26.3])\end{array}$ & $\begin{array}{l}7 / 59(11.9 \% \\
[4.9-22.9])\end{array}$ & $0.496^{b}$ \\
\hline $\mathrm{ALT}, \mathrm{U} / \mathrm{L}$ & Median change [RIQ]; N & $\begin{array}{l}-19.0 \\
{[-58.0,+3.0] ; 61}\end{array}$ & $\begin{array}{l}-20.5[-72.3,+0.5] ; \\
44\end{array}$ & $0.436^{c}$ & & & \\
\hline AST, U/L & Median change [RIQ]; N & $-4[-27,+8] ; 59$ & $-19[-31,+0] ; 43$ & $0.069^{c}$ & & & \\
\hline
\end{tabular}

$n$ : patients with event; N: observed patients. AST, aspartate aminotransferase; ALT, alanine aminotransferase; RIQ, Range interquartile (25th, 75th percentile).

andependent-samples $t$-test.

${ }^{\mathrm{b}}$ Chi-square test.

cMann-Whitney U-test.

to increase the chance of patients accepting to undergo a control biopsy and to confirm this decision at the time the biopsy is being performed, and also to detect whether transaminases exhibit detectable changes along with the changes in liver conditions.

The second limitation of this trial is the dosage we used. We used the same dose $(1000 \mathrm{mg} /$ day $)$ as used in the only available study of metadoxine in NAFLD/NASH,${ }^{13}$ which

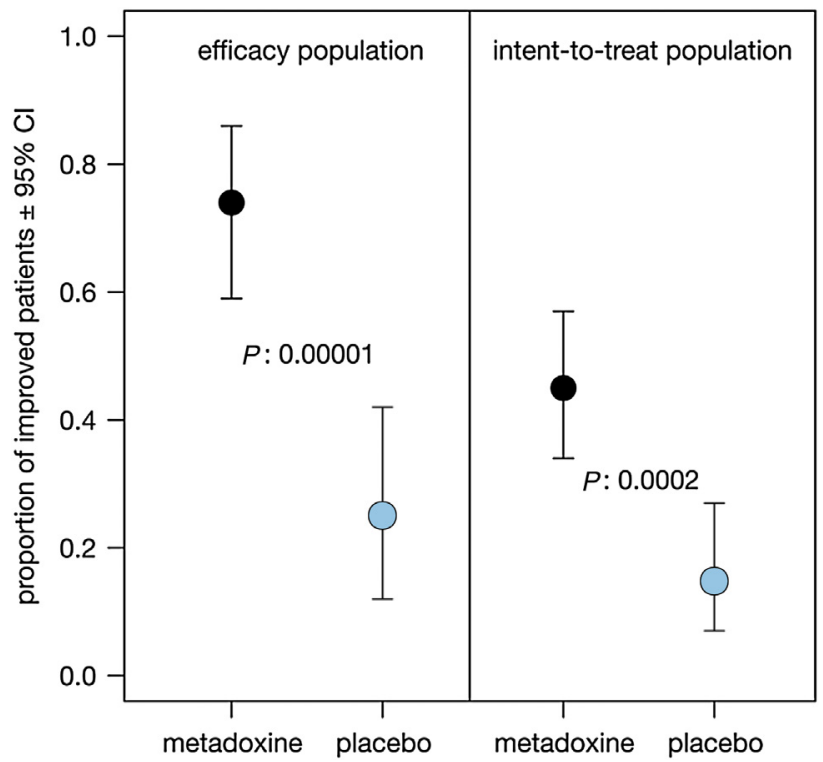

Figure 2 Proportion (with 95\% confidence interval) of patients with improved steatosis score at the end of observation. The $P$-values reported are from the Pearson's chi-square statistic. reported improved symptoms, favorable changes of liver function, improved ultrasonography and improved, but not significantly so, fibrosis score. The study, however, was not comparative. Comparative studies reporting significantly improved ultrasonography/scintigraphy were instead performed on AFLD, using doses of $1.5 \mathrm{~g}^{26,27}$ to $2.0 \mathrm{~g}^{28}$ of metadoxine per day. Since there is no reason to expect that the response in NAFLD/NASH is better than in AFLD/ASH, most likely future trials should be designed with a higher daily dose of metadoxine.

Additionally, the pathology interpretation was not centralized and at each site the biopsies were read by pathologists who have been provided with guidelines for interpretation and there may be inter-reader variability.

In spite of these limitations, however, we could confirm in our study in NASH the ultrasonographic findings reported in the studies in AFLD. Significantly more metadoxine-treated patients $(45.3 \%$ (95\% CI: $34.6 \%-$ $56.6 \%)$ ) exhibited reduced grading of steatosis in comparison with those randomized to placebo $(15.3 \%$ (95\% CI: $8.2 \%-26.5 \%)$ ). The difference in outcome was statistically significant $(P=0.0002)$ in spite of the relatively high number of patients classified as failure because failed or refused to perform the control ultrasonography (39\% of patients equally split across the treatment groups). The size of the difference resulted within the range anticipated in the protocol (30.1\%; 95\% CI: $14.5 \%-43.3 \%)$ and yielded a Number Needed to Benefit of 4 (95\% CI: 3-7).

In conclusion, we confirmed the favorable effect of metadoxine on steatosis also in patients with NASH, 


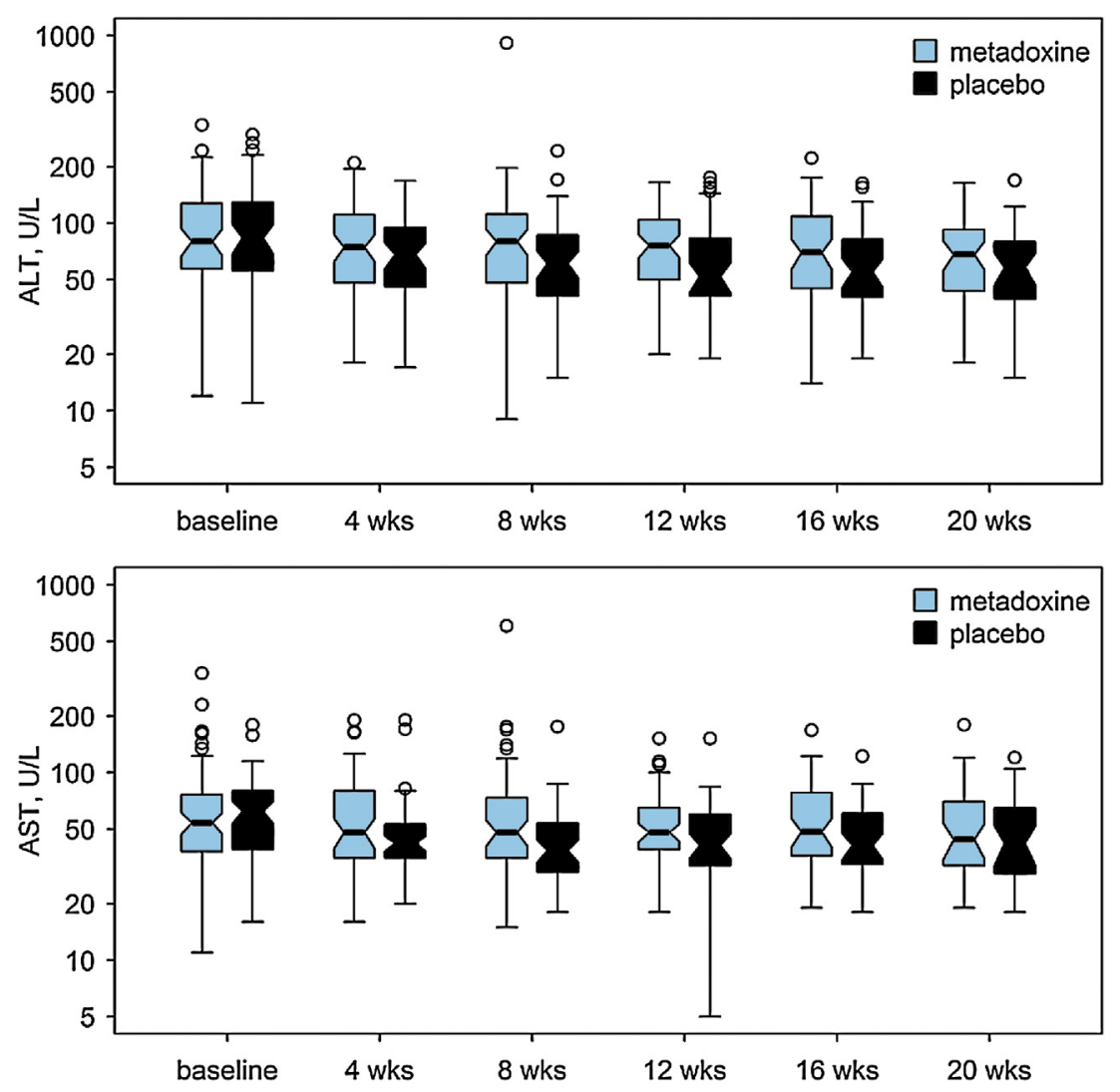

Figure 3 Time course of serum alanine aminotransferase (ALT) and aspartate aminotransferase (AST) shown as box and whisker plot. The thick line indicated the median; the 'hinges' indicate the first and third quartile, the notches approximate the asymptotic 95\% confidence interval around the median, the whiskers extend to the most extreme data point which is no more than 1.5 times the length of the box away from the box; the dots indicate the outliers.

according to the theoretical expectations. ${ }^{29-31}$ In order to confirm the effect on necro-inflammatory and fibrosis scores and the effect on transaminases similar to that seen in alcoholic liver disease, further studies should be performed, possibly with longer treatment of up to one year (also to increase the proportion of patients accepting the control biopsy) and with doses comparable with those used in alcoholic liver disease, i.e. $1500 \mathrm{mg} /$ day.

\section{CONFLICTS OF INTEREST}

The study was sponsored by Eurodrug Laboratories B.V., The Hague, Holland; Dr. Reddy's Laboratories Ltd., Hyderabad, India and Micro Labs Ltd, Bangalore, India. Metadoxine and matching placebo have been supplied by Micro Labs Ltd, Bangalore, India, manufactured under license from Eurodrug Laboratories Ltd.

Bignamini AA received consultancy fees from Eurodrug Laboratories Ltd., Bayer, Alfa-Wassermann.

\section{REFERENCES}

1. Tolman KG, Fonseca V, Dalpiaz A, Tan MH. Spectrum of liver disease in type 2 diabetes and management of patients with diabetes and liver disease. Diabetes Care. 2007;30:734-743.
2. Musso G, Gambino R, Cassader M, Pagano G. A meta-analysis of randomized trials for the treatment of non alcoholic fatty liver disease. Hepatology. 2010;52:79-104.

3. Boettcher E, Csako G, Pucino F, Wesley R, Loomba R. Meta-analysis: pioglitazone improves liver histology and fibrosis in patients with non-alcoholic steatohepatitis. Aliment Pharmacol Ther. 2012;35:66-75.

4. Li W, Zheng L, Sheng C, et al. Systematic review on the treatment of pentoxifylline in patients with non-alcoholic fatty liver disease. Lipids Health Dis. 2011;10:49.

5. Calabrese V, De Bernardis E, Rizza V. Role of metadoxine in the control of the oxidative stress from acute and chronic ethanol intoxication. Boll Soc Ital Biol Sper. 1986;62:1357-1363 [Article in Italian].

6. Calabrese V, Calderone A, Ragusa N, Rizza V. Effects of metadoxine on cellular status of glutathione and of enzymatic defence system following acute ethanol intoxication in rats. Drugs Exp Clin Res. 1996;22:17-24.

7. Calabrese V, Randazzo G, Ragusa N, Rizza V. Long-term ethanol administration enhances age-dependent modulation of redox state in central and peripheral organs of rat: protection by metadoxine. Drugs Exp Clin Res. 1998;24:85-91.

8. Felicioli R, Saracchi I, Flagiello AM, Bartoli C. Effects of pyridoxine-pyrrolidon-carboxylate on hepatic and cerebral ATP levels in ethanol treated rats. Clin Pharmacol Ther Toxicol. 1980;18:177-180.

9. Baldacci M, Catalani R, Bartoli C, Mura U. Effects of piridoxine pyrrolidone-carboxyalte on the liver levels of adenosine triphosphate in rats. Boll Soc Ital Biol Sper. 1982;58:1643-1649 [Article in Italian]. 
10. Arosio B, Santambrogio D, Gagliano N, Annoni G. Changes in expression of the albumin, fibronectin and type I procollagen genes in CCl4-induced liver fibrosis: effect of pyridoxol L, 2-pyrrolidon-5 carboxylate. Pharmacol Toxicol. 1993;73:301-304.

11. Annoni G, Contu L, Tronci MA, Caputo A, Arosio B. Pyridoxol L, 2pyrrolidon-5 carboxylate prevents active fibroplasia in CCl4treated rats. Pharm Res. 1992;25:87-93.

12. Gutiérrez-Ruiz MC, Bucio L, Correa A, et al. Metadoxine prevents damage produced by ethanol and acetaldehyde in hepatocyte and hepatic stellate cells in culture. Pharm Res. 2001;44:431436.

13. Pal'tsev Al, Sharapov IV, Gorbunova EN, et al. Non-alcoholic fatty liver disease: age peculiarities, breakthrough in pathogenetic therapy. Eksp Klin Gastroenterol. 2009;8:19-25 [Article in Russian].

14. Addolorato G, Ancona C, Capristo E, Gasbarrini G. Metadoxine in the treatment of acute and chronic alcoholism: a review. Int J Immunopathol Pharmacol. 2003;16:207-214.

15. Saverymuttu SH, Joseph AEA, Maxwell JD. Ultrasound scanning in the detection of hepatic fibrosis and steatosis. $\mathrm{Br}$ Med J. 1986;292:13-15.

16. Osawa H, Mori Y. Sonographic diagnosis of fatty liver using a histogram technique that compares liver and renal cortical amplitudes. J Clin Ultrasound. 1996;24:25-29.

17. Brunt EM, Janney CG, Di Bisceglie AM, Neuschwander-Tetri BA, Bacon BR. Nonalcoholic steatohepatitis: a proposal for grading and staging the histological lesions. Am J Gastroenterol. 1999;94:2467-2474.

18. Loomba R, Wesley R, Pucino F, et al. Placebo in nonalcoholic steatohepatitis: insight into natural history and implications for future clinical trials. Clin Gastroenterol Hepatol. 2008; 6:1243-1248.

19. Amrapurkar DN, Amrapurkar AD. Nonalcoholic steatohepaitis: clinicopathological profile. J Assoc Physicians India. 2000;48:311313.
20. Agarwal SR, Malhotra V, Sakhuja P, Sarin SK. Clinical, biochemical and histological profile of nonalcoholic steatohepatitis. Indian J Gastroenterol. 2001;20:183-186.

21. Singh SP, Nayak S, Swain M, et al. Prevalence of nonalcoholic fatty liver disease in coastal eastern India: a preliminary ultrasonographic survey. Trop Gastroenterol. 2004;25:76-79.

22. Sanyal AJ. Non-alcoholic fatty liver disease: a medical consequence of globalisation? Indian J Gastroenterol. 2001;20:215-216.

23. Duseja A, Das R, Nanda M, et al. Nonalcoholic steatohepatitis in Asian Indians is neither associated with iron overload nor with HFE gene mutations. World J Gastroenterol. 2005;11:393-395.

24. Le TA, Chen J, Changchien $C$, et al. Effect of colesevelam on liver fat quantified by magnetic resonance in nonalcoholic steatohepatitis: a randomized controlled trial. Hepatology. 2012;56:922-932.

25. Noureddin M, Lam J, Peterson MR, et al. Utility of magnetic resonance imaging versus histology for quantifying changes in liver fat in nonalcoholic fatty liver disease trials. Hepatology. 2013;58:1930-1940.

26. Caballería J, Parés A, Brú C, et al. Metadoxine accelerates fatty liver recovery in alcoholic patients: results of a randomized double-blind, placebo-control trial. J Hepatol. 1998;28:54-60.

27. Malhotra PS, Singh BR, Narotam B, Kaur KP. A study of metadoxine in alcoholic liver disease. J Assoc Physicians India. 2005;53:352-353.

28. Vedrova NN, Gnezdilova Nlu. Metadoxyl in combined treatment of alcohol damage to the liver. Klin Med Mosk. 2001;79:56-58 [Article in Russian].

29. Fehér J, Lengyel G. A new approach to drug therapy in non-alcoholic steatohepatitis (NASH). J Int Med Res. 2003;31:437-451.

30. Váli L, Blázovics A, Fehér J. The therapeutic effect of metadoxine on alcoholic and non-alcoholic steatohepatitis. Orv Hetil. 2005;146:2409-2414 [Article in Hungarian].

31. Fehér J, Váli L, Blázovics A, Lengyel G. The Beneficial effect of metadoxine (pyridoxine-pyrrolidone-carboxylate) in the treatment of fatty liver diseases. CEMED. 2009;3:65-76. 